\title{
Differential SPECT Activation Patterns Associated with PASAT Performance May Indicate Frontocerebellar Functional Dissociation in Chronic Mild Traumatic Brain Injury
}

\author{
Naoya Hattori ${ }^{1}$, Megan $\mathrm{Swan}^{2}$, Gary A. Stobbe ${ }^{3}$, Jay M. Uomoto ${ }^{4}$, Satoshi Minoshima ${ }^{1}$, David Djang ${ }^{5}$, \\ Ruben Krishnananthan ${ }^{1}$, and David H. Lewis ${ }^{1}$ \\ ${ }^{I}$ Department of Radiology, University of Washington School of Medicine, Seattle, Washington; ${ }^{2}$ Department of Psychology, Seattle \\ Pacific University, Seattle, Washington; ${ }^{3}$ Department of Neurology, University of Washington School of Medicine, Seattle, \\ Washington; ${ }^{4}$ Department of Neuropsychology, Center for Polytrauma Care, VA Puget Sound Health Care System, Seattle, \\ Washington; and ${ }^{5}$ Department of Nuclear Medicine, Seattle Nuclear Medicine, Seattle, Washington
}

Patients with mild traumatic brain injury (TBI) often complain of cognitive fatigue during the chronic recovery phase. The Paced Auditory Serial Addition Test (PASAT) is a complex psychologic measure that may demonstrate subtle deficiencies in higher cognitive functions. The purpose of this study was to investigate the brain activation of regional cerebral blood flow (rCBF) with PASAT in patients with mild TBI to explore mechanisms for the cognitive fatigue. Methods: Two groups consisting of 15 patients with mild TBI and 15 healthy control subjects underwent 99mTc-ethylene cysteine dimer SPECT at rest and during PASAT on a separate day. Cortical rCBF was extracted using a 3-dimensional stereotactic surface projection and statistically analyzed to identify areas of activation, which were compared with PASAT performance scores. Results: Image analysis demonstrated a difference in the pattern of activation between patients with mild TBI and healthy control subjects. Healthy control subjects activated the superior temporal cortex (Brodmann area [BA] 22) bilaterally, the precentral gyrus (BA 9) on the left, and the precentral gyrus (BA 6) and cerebellum bilaterally. Patients with mild TBI demonstrated a larger area of supratentorial activation (BAs $9,10,13$, and 46 ) but a smaller area of activation in the cerebellum, indicating frontocerebellar dissociation. Conclusion: $\mathrm{Pa}-$ tients with mild $\mathrm{TBI}$ and cognitive fatigue demonstrated a different pattern of activation during PASAT. Frontocerebellar dissociation may explain cognitive impairment and cognitive fatigue in the chronic recovery phase of mild traumatic brain injury. Key Words: traumatic brain injury; cognitive fatigue; SPECT; activation study; PASAT

J Nucl Med 2009; 50:1054-1061

DOI: 10.2967/jnumed.108.060368

Received Dec. 3, 2008; revision accepted Mar. 17, 2009.

For correspondence or reprints contact: Naoya Hattori, Nuclear Medicine, Radiology, University of Washington, Box 356113, 1959 NE Pacific St., Seattle, WA 98195.

E-mail: nhattori@u.washington.edu

COPYRIGHT ๑ 2009 by the Society of Nuclear Medicine, Inc.
$\mathbf{P}$ atients with mild traumatic brain injury (TBI) experience psychologic and cognitive problems acutely after injury, including cognitive slowing, poor concentration, attention difficulties, and impaired memory. These symptoms gradually resolve over time in most. However, complex psychologic measures may demonstrate subtle deficiencies in higher cognitive functions for months or years after TBI (1).

The Paced Auditory Serial Addition Test (PASAT) is one of the tests most frequently used by neuropsychologists to assess attentional processing. It was developed as an experimental tool to examine immediate (i.e., working) memory and attention and has been clinically applied to examine the effects of TBI (2-7). In healthy subjects, PASAT has been reported to activate mainly the frontal and parietal cortex, with limited interindividual variation (8). Despite a large number of investigations using PASAT as a tool of neuropsychologic measurement, only a few imaging studies have investigated the activation pattern of PASAT in patients with TBI. Christodoulou et al. investigated the activation pattern using functional MRI in patients with moderate to severe TBI and reported a pattern similar to that in healthy subjects although activation was more lateralized toward the right hemisphere (9). Until now, the activation pattern of mild TBI has not been investigated.

The purpose of this study was to investigate the activation pattern with PASAT in patients with mild TBI and persistent cognitive complaints, specifically the complaint of cognitive fatigue.

\section{MATERIALS AND METHODS}

\section{Study Design}

The study was reviewed and approved by the Human Subjects Committee at the University of Washington and Seattle Pacific 
University. Participants gave written informed consent. Each participant underwent SPECT twice on a separate day $1 \mathrm{wk}$ apart.

As a control, the resting scan was done first. Participants were asked to close their eyes and rest or to use an eye mask to assist them in keeping their eyes closed. Participants were not instructed to think of anything in particular and were discouraged from speaking. Their ears were unplugged and were exposed to the lowlevel ambient noise in the area so that auditory stimulation was present. The SPECT scan was started 45 min after the injection of $1,110 \mathrm{MBq}$ of ${ }^{99 \mathrm{~m}} \mathrm{Tc}-$ labeled ethylene cysteine dimer (Neurolite; DuPont).

The activation scan was completed $1 \mathrm{wk}$ after the rest scan. All procedures were the same except that, instead of sitting quietly, the participants completed PASAT at the time of the tracer injection. PASAT is a complex test of sustained divided attention. Respondents listen to a series of single-digit numbers on a tape and are required to add each number to each preceding one to give a verbal response. There are 4 separate trials of 50 digits each, with the starting presentation speed of 1 digit every $2.4 \mathrm{~s}$ (trial 1 ) increasing to 1 digit every $2.0 \mathrm{~s}$ (trial 2), 1 every $1.8 \mathrm{~s}$ (trial 3), and 1 every $1.6 \mathrm{~s}$ (trial 4). The test takes into account one's speed and accuracy of controlled information processing and the effects of an increasing load (10).

Before the start of PASAT during the experimental scan, the research psychometrist aurally administered to the participant a 10 -s practice trial to serve as a primer. The injection took place 11 digits $(24 \mathrm{~s})$ into the first trial so that the cerebral radiotracer uptake, which occurs from $20 \mathrm{~s}$ to $1.5 \mathrm{~min}$, would be accomplished during the remaining 2 min $6 \mathrm{~s}$ required for completion of the trial. PASAT was scored for the total number of correct responses by trial and across trials. Scoring took approximately 10 min for all 4 trials. As with the rest scan, the SPECT scan started $45 \mathrm{~min}$ after injection.

\section{Subjects}

The patient group included 15 subjects who had sustained a mild TBI. Diagnosis was based on the American Congress of Rehabilitation Medicine criterion for a mild traumatic brain injury (11). All patients reported loss of consciousness for less than $30 \mathrm{~min}$ and posttraumatic amnesia for less than $24 \mathrm{~h}$. Each of these patients had been referred for brain SPECT by a neurologist as a part of routine clinical neurologic evaluations as described in the 2007 guideline of the American College of Radiology (12). Patients were selected if they sustained a closed head injury more than 6 mo before the brain SPECT scan; complained to the examining neurologist of cognitive fatigue, defined as the inability to maintain attention and concentration during a sustained task; had medical charts showing no diagnosis of major depressive disorder or had a Beck Depression Inventory score of less than 14; and had medical charts showing no comorbid medical disorder with fatigue-related symptoms (e.g., multiple sclerosis; fibromyalgia; chronic fatigue syndrome; systemic or central lupus; or hypothyroidism). None of the participants with mild TBI had abnormal results on anatomic imaging (CT or MRI), nor had any participants a skull fracture. All patients underwent a series of neuropsychologic tests including Positive and Negative Affect Scale (PANAS); Borg Perception of Mental Exertion; Neuropsychological Impairment Scale, providing T-scores for global, affective, cognitive, attention, and memory components; and Fatigue Severity Scale. In addition, the results of their original neuropsychologic test were reviewed.

Potential healthy participants were recruited from responders to advertisements or recruitment posters. All were briefly screened by telephone for medical history, neuropsychologic trauma (e.g., loss of consciousness, exposure to chemicals, or head injury), and alcohol use; underwent a physical examination; and had a computer-assisted structured psychiatric diagnostic interview, the Diagnostic Interview Schedule, version III-A (13). Those who reported chronic fatigue or fibromyalgia, had a major medical illness (such as high blood pressure, diabetes, or severe asthma), or had any current or historic psychiatric diagnosis (including alcohol or drug abuse) were excluded from the study population. Only right-handed subjects were included in the study.

\section{SPECT Image Processing}

SPECT was performed using either of 2 Phillips $\gamma$-cameras: a Prism 3000 triple-head with ultra-high-resolution fanbeam collimators or an ADAC Genesys Epic dual-head with low-energy high-resolution collimators.

Both systems reconstructed SPECT images using filtered backprojection with Chang attenuation correction. They yielded approximately 40 transverse slices obtained at single-pixel thickness ( $2 \mathrm{~mm}$ ) with a system spatial resolution of $7 \mathrm{~mm}$ (full width at half-maximum). Images were analyzed with the Neurostat software package (University of Washington) $(14,15)$. Each pair of activation and resting images was spatially coregistered first with a rigid transformation algorithm, followed by stereotactic spatial normalization to the Talairach atlas with a nonlinear transformation algorithm. Spatially normalized resting and activation images were averaged to produce a single-template image. Finally, individual resting and activation images were processed for a pixelwise normalization of global counts using the template image.

\section{Data and Statistical Analysis}

After image registration and pixel normalization, individual activation and resting images were compared to investigate regional activation and suppression with PASAT. Statistical analysis was done using a paired $t$ test on a voxel-by-voxel basis. A total of 118,787 voxels were analyzed. To visually investigate regions showing activation or suppression during PASAT, we highlighted voxels showing activation $(z$ score $>1.96, P<0.05)$ or suppression $(z$ score $<-1.96, P<0.05)$ on a template MR image. In addition, regions showing activation or suppression were investigated using the Talairach atlas, based on the coordinates of voxels and Brodmann areas (BAs) on the template image. The level of significance was set at $P<0.05$, corresponding to a $z$ score of 1.96. After correction of multiple comparisons, $P<0.05$ corresponds to a $z$ score of 4.50 . For correlation analysis, pairwise subtractions were conducted between resting and activated scans. Cortical activity after the individual subtraction was extracted using 3-dimensional stereotactic surface projection and mapped on the brain surface consisting of a total of 15,964 points. The subtracted cortical activity in each voxel was compared with PASAT score. Cortical regions showing a significant correlation with PASAT score were investigated using coordinates of subtraction analysis and Talairach atlas. The level of significance was set at $P<0.0001$. PASAT scores were analyzed using an unpaired $t$ test. The level of significance was set at $P<0.05$ for the comparison of all trials of PASAT scores.

\section{RESULTS}

The final study population included 15 patients with mild TBI and 15 healthy control subjects. The mild-TBI group consisted of 12 women and 3 men, with a mean age ( \pm SD) 
of $45 \pm 11 \mathrm{y}$ and an age range of $27-60 \mathrm{y}$. Thirteen of the patients had been injured as a passenger in a motor vehicle accident, and 2 had been injured as a bicycle rider in a collision with a motor vehicle. The patients had an average of $16 \pm 3 \mathrm{y}$ of education (range, 12-24 y). The average interval between the incident and the SPECT scan was 28.6 mo. The results (mean $\pm \mathrm{SD}$ ) of the psychiatric interviews were as follows: mean total scores of $17.5 \pm 2.6$ for Borg Perception of Mental Exertion, $54.4 \pm 7.7$ for Fatigue Severity Scale. The mean T-scores for the Neuropsychological Impairment Scale were 73.1 \pm 6.2 for global impairment, $60.9 \pm 5.7$ for affective, $73.3 \pm 6.1$ for cognitive efficiency, $71.8 \pm 7.6$ for attention, and $66.9 \pm 6.4$ for memory. The original cohort was 16 patients; 1 patient was excluded because of movement during brain SPECT. No patient was excluded because of depression. Six of the 15 were involved in litigation at the time of the procedure. None were involved in disability claims. The healthy control group consisted of 12 women and 3 men. This group did not significantly differ from the patients in age $(43 \pm 9 \mathrm{y}$, ranging from 28 to $58 \mathrm{y})$ or years of education $(17 \pm 2 \mathrm{y}$, ranging from 14 to $20 \mathrm{y}$ ).

In all 4 trials, patients with mild TBI had lower PASAT scores than did the control subjects. PASAT results (patients vs. controls) were $35.2 \pm 13.0$ vs. $43.7 \pm 3.7(P<0.05)$ for trial $1,34.2 \pm 10.6$ vs. $39.1 \pm 6.3(P=$ not statistically significant $[\mathrm{NS}])$ for trial $2,30.9 \pm 11.8$ vs. $35.3 \pm 6.8$ $(P=\mathrm{NS})$ for trial 3 , and $24.6 \pm 11.8$ vs. $28.7 \pm 9.1(P=$ NS) for trial 4 . Among the 4 trials, the score of the first trial of PASAT (PASAT24, performed during the ${ }^{99 \mathrm{~m} T c-e t h y l e n e}$ cysteine dimer uptake period) was significantly lower for patients with mild TBI. The result of PASAT24 did not correlate with population age or years of education in either of the groups.

Figure 1 compares increased regional cerebral blood flow (rCBF) during the PASAT activation task between the 2 groups. Pixels showing a $z$ score greater than 1.96,

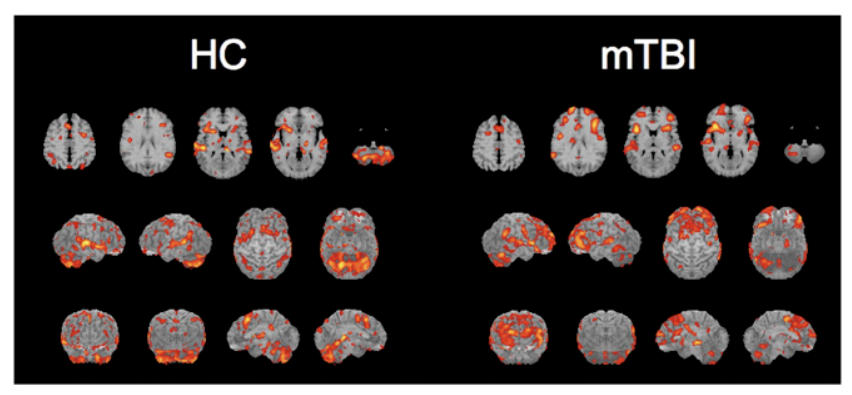

FIGURE 1. Comparison of activated rCBF during PASAT task between healthy control subjects $(\mathrm{HC})$ and patients with mild TBI (mTBI). Results of $z$ score analyses are overlaid on MR image of template atlas. Voxels showing $z$ score $>1.96$ are highlighted. First row: representative 5 slices. Second and third rows: surface projection map images of same results.

corresponding to a $P$ value of less than 0.05 , are highlighted. Tables 1 and 2 summarize regional activation in healthy control subjects and patients with mild TBI, respectively. In healthy control subjects, the PASAT task activated the superior temporal cortex (BA 22) bilaterally, the precentral gyrus (BA 9) on the left, and the precentral gyrus (BA 6) and cerebellum bilaterally. The anterior cingulate cortex showed activation but with a relatively low $z$ score $(2.88, P<0.01)$ (Table 1). Patients with mild TBI also had bilateral activation of the superior temporal cortex (BA 22) and precentral gyrus (BA 6). However, they demonstrated larger areas of bilateral activation in the dorsolateral prefrontal cortex (BAs 9, 10, and 46) and insula (BA 13), whereas smaller areas of activation were seen in cerebellum (Table 2).

Figure 2 compares suppressed rCBF during the PASAT activation task. Tables 3 and 4 summarize regional suppression in healthy control subjects and patients with mild TBI, respectively. In healthy control subjects, the PASAT task suppressed areas of the medial frontal to anterior cingulate

\begin{tabular}{|c|c|c|c|c|}
\hline \multirow[b]{2}{*}{$z$ score } & \multicolumn{3}{|c|}{ Talairach coordinates (mm) } & \multirow[b]{2}{*}{ Region } \\
\hline & $x$ & $y$ & $z$ & \\
\hline 5.24 & 33 & 5 & 36 & L precentral gyrus, BA 9 \\
\hline 4.52 & -64 & -22 & 4 & R superior temporal gyrus, BA 22 \\
\hline 4.43 & 37 & -71 & -43 & L cerebellum \\
\hline 4.38 & 10 & -33 & 9 & $\mathrm{~L}$ thalamus \\
\hline 4.32 & -1 & 21 & 50 & R superior frontal gyrus, BA 8 \\
\hline 4.27 & -19 & -62 & -40 & $\mathrm{R}$ cerebellum \\
\hline 3.87 & -24 & 14 & 0 & R putamen \\
\hline 3.68 & 62 & -35 & 9 & L superior temporal gyrus, BA 22 \\
\hline 3.4 & -37 & 17 & 11 & $R$ insula, BA 13 \\
\hline 3.38 & -35 & 23 & 4 & $\mathrm{R}$ inferior frontal gyrus, BA 45 \\
\hline 3.36 & -42 & -15 & 27 & R precentral gyrus, BA 6 \\
\hline 3.29 & -8 & -13 & 11 & $\mathrm{R}$ thalamus \\
\hline 3.12 & 3 & 1 & 52 & L medial frontal gyrus, BA 6 \\
\hline
\end{tabular}




\section{TABLE 2. Summary of Activation in Mild TBI}

\begin{tabular}{|c|c|c|c|c|}
\hline \multirow[b]{2}{*}{ z score } & \multicolumn{3}{|c|}{ Talairach coordinates (mm) } & \multirow[b]{2}{*}{ Region } \\
\hline & $x$ & $y$ & $z$ & \\
\hline 4.67 & -37 & 17 & 4 & $\mathrm{R}$ insula, BA 13 \\
\hline 4.03 & 39 & 26 & 20 & $\mathrm{~L}$ middle frontal gyrus, BA 46 \\
\hline 4.00 & -8 & -26 & -2 & $\mathrm{R}$ thalamus \\
\hline 3.85 & -17 & 62 & 22 & R superior frontal gyrus, BA 10 \\
\hline 3.82 & -42 & -10 & 32 & R precentral gyrus, BA 6 \\
\hline 3.80 & 35 & 17 & 11 & L insula, BA 13 \\
\hline 3.62 & -53 & -53 & -22 & $\mathrm{R}$ cerebellum \\
\hline 3.57 & 39 & 37 & 4 & $\mathrm{~L}$ inferior frontal gyrus, BA 46 \\
\hline 3.55 & 35 & -6 & 32 & L precentral gyrus, BA 6 \\
\hline 3.54 & -42 & -1 & 20 & $\mathrm{R}$ inferior frontal gyrus, BA 9 \\
\hline 3.43 & 53 & -28 & 9 & L superior temporal gyrus, BA 41 \\
\hline 3.36 & -62 & -46 & 22 & R supramarginal gyrus, BA 40 \\
\hline 3.34 & -19 & 5 & -2 & R putamen \\
\hline 3.33 & 19 & 55 & 25 & L superior frontal gyrus, BA 9 \\
\hline 3.31 & -28 & 26 & 32 & $\mathrm{R}$ middle frontal gyrus, BA 9 \\
\hline 3.29 & -51 & -17 & 4 & R superior temporal gyrus, BA 22 \\
\hline 3.18 & 21 & -1 & -2 & L putamen \\
\hline
\end{tabular}

cortex (BAs 9, 10, and 24) bilaterally, the parahippocampal gyrus (BA 19) bilaterally, the posterior cingulate cortex (BA 23) on the left, and the precuneus (BA 31) on the right (Table 3). Patients with mild TBI showed suppression in the left posterior cingulate cortex (BA 29) and right precuneus (BA 7). However, they showed less suppression in the medial frontal cortex and larger areas of suppression in the parietooccipital cortex (BAs 7, 18, and 19) (Table 4).

Figure 3 shows regions with a significant positive correlation between activation and PASAT24 scores. Tables 5 and 6 summarize the regional correlation in healthy control subjects and patients with mild TBI, respectively. In healthy control subjects, PASAT24 scores correlated significantly with activation in the right and left cerebellum, right superior to middle frontal gyrus (BA 6), left inferior frontal cortex (BA 47), and right superior temporal cortex

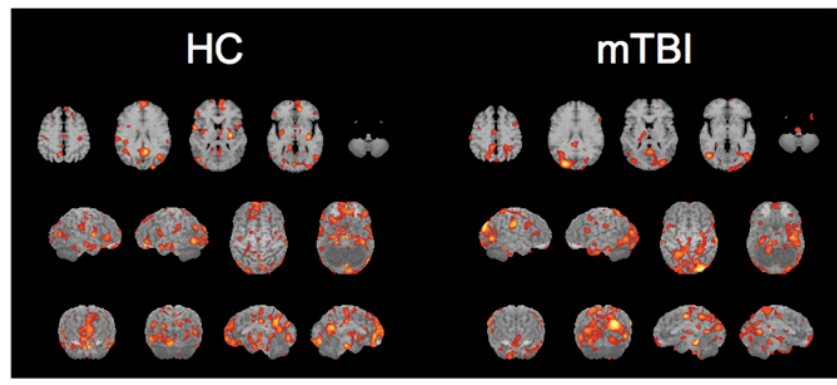

FIGURE 2. Comparison of suppressed rCBF during PASAT task between healthy control subjects $(\mathrm{HC})$ and patients with mild TBI (mTBI). Results of $z$ score analyses are overlaid on MR image of template atlas. Voxels showing $z$ score $>$ 1.96 are highlighted. First row: representative 5 slices. Second and third rows: surface projection map images of same results.
(BA 22). Patients with mild TBI showed less correlation in the cerebellum, whereas similar activations were observed in the superior temporal cortex (BA 22) and superior frontal cortex (BA 6). Additional areas of correlation were found in the dorsolateral prefrontal cortex (BAs 45 and 46) and anterior cingulate cortex (BA 32) (Tables 5 and 6).

\section{DISCUSSION}

PASAT results in the present study showed a tendency toward lower PASAT scores in mild-TBI patients than in control subjects. In particular, the score of the first trial of PASAT (PASAT24) was significantly lower in patients with mild TBI who complained of cognitive fatigue.

TBI is not rare. Of all cases, mild TBI comprises approximately $80 \%$. On the basis of the U.S. annual incidence of mild TBI (503.1 cases per 100,000 individuals (16)) and the percentage of mild-TBI cases that are symptomatic after a year $(10 \%(1))$, the incidence of chronic symptomatic mild TBI is calculated at approximately 50 cases per 100,000 individuals. This number is higher than the incidence of Parkinson disease, reported to be 19.0 per 100,000 (17). In addition, the relatively younger cohort in mild TBI potentially faces decades of disability (1). Symptoms after mild TBI can be physical, cognitive, or social. Most neuropsychologic symptoms of mild TBI generally clear completely within the first 3 mo (1). Among these symptoms, fatigue is one of the most frequently reported, accounting for almost one third of a large group of mildTBI patients 6 mo after injury, and is associated with limitations in daily functioning (18). In the present study, mild-TBI patients were recruited from a population that presented with cognitive fatigue as a prominent contributor to their inability to return to normal work despite relatively 
TABLE 3. Summary of Suppression in Healthy Control Subjects

\begin{tabular}{|c|c|c|c|c|}
\hline \multirow[b]{2}{*}{ z score } & \multicolumn{3}{|c|}{ Talairach coordinates (mm) } & \multirow[b]{2}{*}{ Region } \\
\hline & $x$ & $y$ & $z$ & \\
\hline-4.86 & 30 & -17 & 4 & L putamen \\
\hline-4.69 & 6 & -53 & 20 & L posterior cingulate, BA 23 \\
\hline-4.14 & -26 & -51 & -7 & R parahippocampal gyrus, BA 19 \\
\hline-4.08 & 1 & -19 & -20 & Pons \\
\hline-3.99 & 37 & -44 & -7 & L parahippocampal gyrus, BA 19 \\
\hline-3.94 & -10 & -51 & 32 & R precuneus, BA 31 \\
\hline-3.83 & 8 & -82 & -9 & L lingual gyrus, BA 18 \\
\hline-3.82 & 12 & 48 & 0 & $\mathrm{~L}$ anterior cingulate, BA 10 \\
\hline-3.73 & 48 & -64 & -4 & L inferior temporal gyrus, BA 37 \\
\hline-3.65 & -1 & -17 & 36 & $R$ cingulate gyrus, BA 24 \\
\hline-3.58 & -53 & 3 & 9 & R precentral gyrus, BA 6 \\
\hline-3.44 & -1 & -80 & -4 & $\mathrm{R}$ lingual gyrus, BA 18 \\
\hline-3.35 & 42 & -26 & -22 & L fusiform gyrus, BA 20 \\
\hline-3.33 & -8 & 62 & -4 & R medial frontal gyrus, BA 10 \\
\hline-3.29 & 6 & 55 & 14 & L medial frontal gyrus, BA 9 \\
\hline-3.28 & -42 & -31 & 29 & $\mathrm{R}$ inferior parietal lobule, BA 40 \\
\hline-3.28 & -26 & 23 & -14 & $\mathrm{R}$ inferior frontal gyrus, BA 47 \\
\hline-3.23 & 39 & -64 & 25 & L middle temporal gyrus, BA 39 \\
\hline-3.23 & 24 & -89 & 22 & L middle occipital gyrus, BA 18 \\
\hline-3.22 & 48 & -13 & -9 & L superior temporal gyrus, BA 22 \\
\hline-3.22 & -1 & -60 & 18 & R precuneus, BA 23 \\
\hline-3.17 & -46 & -71 & 9 & R middle occipital gyrus, BA 19 \\
\hline-3.14 & -17 & -1 & -25 & $\mathrm{R}$ uncus, BA 28 \\
\hline-3.12 & -57 & -15 & -11 & R middle temporal gyrus, BA 21 \\
\hline-3.1 & -10 & -78 & 25 & R cuneus, BA 18 \\
\hline
\end{tabular}

normal neuropsychologic test results. Subjectively, however, they experienced significant levels of perceived global impairment, attention and memory problems, higher-order mental functioning problems, and depression and anxiety (affect scale). The selection process may have been biased by the fact that most of the present cohort was of high intelligence and had cognitively demanding jobs: 3 registered nurses, 1 physician, 1 attorney, 3 executives, 1 social worker, 1 grief counselor, 1 contractor, 2 homemakers, 1 office manager, and 1 computer programmer. Other possible causes of selection bias are the fact that all patients were in the chronic phase of TBI, all had been injured more

\section{TABLE 4. Summary of Suppression in Mild TBI}

\begin{tabular}{|c|c|c|c|c|}
\hline \multirow[b]{2}{*}{ z score } & \multicolumn{3}{|c|}{ Talairach coordinates (mm) } & \multirow[b]{2}{*}{ Region } \\
\hline & $x$ & $y$ & $z$ & \\
\hline-4.82 & -26 & -80 & 25 & R cuneus, BA 19 \\
\hline-4.41 & -8 & -31 & -14 & $\mathrm{R}$ cerebellum \\
\hline-4.19 & -3 & -6 & 47 & R cingulate gyrus, BA 24 \\
\hline-3.94 & -60 & -19 & 34 & R postcentral gyrus, BA 3 \\
\hline-3.75 & -44 & -64 & 2 & $\mathrm{R}$ inferior temporal gyrus, BA 37 \\
\hline-3.72 & 1 & -55 & 11 & L posterior cingulate, BA 29 \\
\hline-3.60 & 24 & -76 & -7 & L lingual gyrus, BA 18 \\
\hline-3.59 & 39 & -13 & -22 & L subgyral, BA 20 \\
\hline-3.46 & -10 & -42 & 45 & R precuneus, BA 7 \\
\hline-3.44 & -35 & -60 & 18 & $\mathrm{R}$ middle temporal gyrus, BA 19 \\
\hline-3.39 & 33 & -76 & 14 & L middle occipital gyrus, BA 19 \\
\hline-3.29 & 24 & -53 & 56 & L superior parietal lobule, BA 7 \\
\hline-3.25 & 46 & -69 & 0 & L inferior temporal gyrus, BA 37 \\
\hline-3.22 & -28 & -58 & -4 & R parahippocampal gyrus, BA 19 \\
\hline-3.18 & -39 & -55 & 18 & R superior temporal gyrus, BA 22 \\
\hline-3.18 & -39 & -42 & -16 & $R$ fusiform gyrus, BA 37 \\
\hline
\end{tabular}




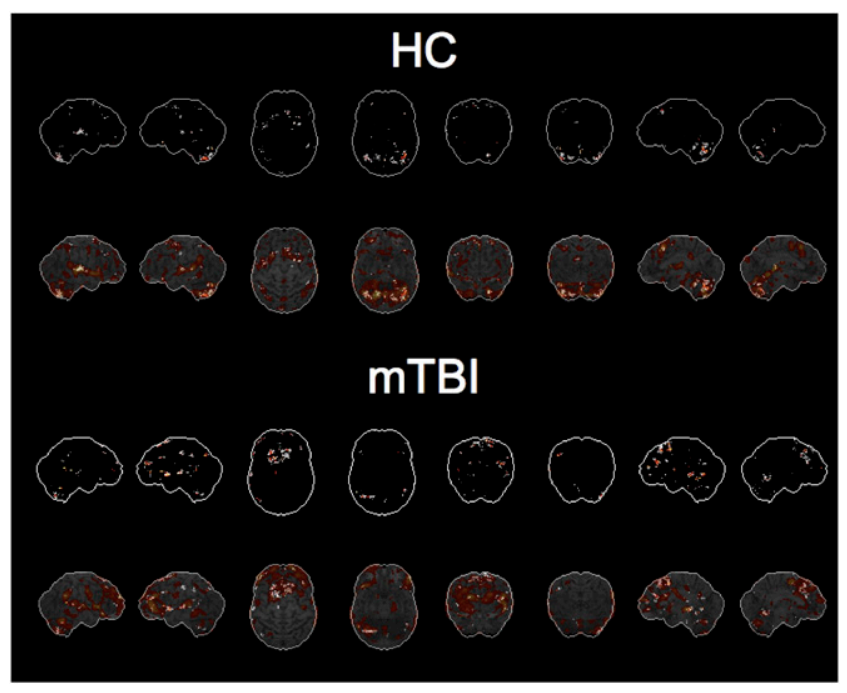

FIGURE 3. Results of correlation analyses. First and third rows show voxels with positive correlation between regional activation and PASAT2 4 scores $(z$ score $>1.96)$ in healthy control subjects $(\mathrm{HC})$ and patients with mild TBI (mTBI), respectively. Second and fourth rows show same results overlaid on corresponding activation maps.

than 6 mo previously, all had mild TBI, all had normal CT or MRI results, none had a primary psychiatric condition, and all were of middle age (chosen as such for better matching). In short, the present study focused on cognitive fatigue in a group of patients with chronic, mild TBI and relatively normal neuropsychologic test results. This selection bias might lead to PASAT results showing only mild neuropsychologic impairment. In the present study, patients with mild TBI demonstrated reproducibly lower scores, but the differences reached statistical significance only in the first of the 4 sessions.

Statistical analyses of rCBF demonstrated a different pattern of activation between patients with mild TBI and healthy control subjects. The activation task induced a dynamic change in supratentorial $\mathrm{rCBF}$ in mild-TBI patients, whereas negligible activation was observed in the cerebellum. The PASAT activation task is conceptualized as multifactorial. It requires the successful completion of numerous cognitive functions, including executive control, numeric addition, attention, and working memory $(19,20)$, which are expected to induce multiple areas of activation. Our results, showing activation in the dorsolateral prefrontal cortex, right and left superior temporal gyrus, right and left medial frontal cortex, and cerebellum in healthy control subjects, are consistent with the above hypothesis. This distribution is in agreement with previous studies that reported a similar regional activation pattern with PASAT in healthy control subjects $(8,21)$. We observed a pattern of activation in mild-TBI patients different from that in healthy control subjects. Patients with mild TBI presented with less activation in the cerebellum despite relatively more activation in the prefrontal cortex. This functional frontocerebellar dissociation may indicate impaired connection fibers due to axonal injury or degeneration after TBI. However, the present results are difficult to interpret because of the lack of evidence of, or the absence of, structural abnormalities in white matter. Future investigations should include newer techniques such as diffusion tensor tractography, which allows white matter connections to be assessed in detail even in patients without an anatomic abnormality on conventional CT or MRI. The mechanism of frontocerebellar dissociation is also suggested by animal experiments that have reported axonal fiber track degeneration in the cerebellum, particularly in the inferior vermis, of every injured animal even though the cortex had been the target of focal injury (22). Interestingly, a recent clinical investigation on children with mild to moderate TBI reported significantly reduced white matter volumes in the cerebellum and its projection areas, including the dorsolateral prefrontal cortex, thalamus, and pons (23). The projection areas in patients with mild TBI showed greater activation in the present study, particularly in the dorsolateral prefrontal cortex, corresponding to the areas of working memory and executive functions (24). This finding may indicate preserved gray matter that was activated to compensate for the frontocerebellar circuit (25). Such compensatory activation was previously reported in alcoholics using functional MRI (26). Their pattern of activation was slightly different from that in mild-TBI patients, probably because white matter connection fibers were not

\begin{tabular}{|c|c|c|c|c|c|}
\hline \multirow[b]{2}{*}{$R$} & \multirow[b]{2}{*}{ z score } & \multicolumn{3}{|c|}{ Talairach coordinates (mm) } & \multirow[b]{2}{*}{ Region } \\
\hline & & $x$ & $y$ & $z$ & \\
\hline 0.979806 & 3.43 & -2 & -59 & -25 & $\mathrm{R}$ cerebellum \\
\hline 0.949166 & 3.27 & 38 & -72 & -27 & L cerebellum \\
\hline 0.926906 & 2.66 & -23 & 7 & 63 & R superior frontal gyrus, BA 6 \\
\hline 0.918788 & 2.59 & 36 & 27 & -16 & L inferior frontal gyrus, BA 47 \\
\hline 0.885989 & 2.35 & -32 & 20 & 54 & R middle frontal gyrus, BA 6 \\
\hline 0.849835 & 2.14 & -65 & -27 & 5 & R superior temporal gyrus, BA 22 \\
\hline 0.844485 & 2.12 & 0 & -29 & 9 & $\mathrm{R}$ thalamus \\
\hline
\end{tabular}


TABLE 6. Summary of Correlation with PASAT in Mild TBI

\begin{tabular}{|c|c|c|c|c|c|}
\hline \multirow[b]{2}{*}{$R$} & \multirow[b]{2}{*}{ z score } & \multicolumn{3}{|c|}{ Talairach coordinates (mm) } & \multirow[b]{2}{*}{ Region } \\
\hline & & $x$ & $y$ & $z$ & \\
\hline 0.970935 & 3.23 & 9 & 16 & 65 & L superior frontal gyrus, BA 6 \\
\hline 0.959598 & 3.03 & 52 & 23 & 5 & L inferior frontal gyrus, BA 45 \\
\hline 0.947813 & 2.88 & 52 & 11 & -2 & L superior temporal gyrus, BA 22 \\
\hline 0.939844 & 2.79 & -2 & 11 & 50 & R superior frontal gyrus, BA 6 \\
\hline 0.934855 & 2.74 & -65 & -45 & 11 & R superior temporal gyrus, BA 22 \\
\hline 0.881045 & 2.62 & -2 & -56 & 0 & $\mathrm{R}$ cerebellum \\
\hline 0.777861 & 2.27 & -2 & 36 & 20 & $\mathrm{R}$ anterior cingulate, BA 32 \\
\hline 0.856461 & 2.18 & 47 & -65 & 36 & $\mathrm{~L}$ angular gyrus, BA 39 \\
\hline 0.850716 & 2.15 & 52 & 45 & 5 & L middle frontal gyrus, BA 46 \\
\hline
\end{tabular}

damaged in the alcoholic population without a history of brain trauma.

Finally, we observed in controls that activation in the cerebellar cortex correlated significantly with PASAT performance score. Although patients with mild TBI showed less correlation in the cerebellum, they showed significant correlations in the inferior frontal and superior temporal cortices instead. This finding is consistent with the hypothesis suggesting a mechanism of compensation. The results of the present study contrast with previous activation studies on depressed populations. Those studies have generally shown less activation in prefrontal cortices during neuropsychologic tasks $(27,28)$. Depressed patients may be less interested in participating and may not make an effort, which is needed to activate the compensatory mechanism involving large areas of the prefrontal cortices. In the present study, however, the mild-TBI subjects clearly made an effort, as the overall PASAT results were not statistically worse than those of healthy controls although PASAT is challenging. Healthy control subjects had less evident correlations in prefrontal regions, probably because of intact cerebellar function and an efficient frontocerebellar circuit.

The major methodologic limitations of the present study include the lack of a group comparison of baseline or activation rCBF between healthy control subjects and patients with mild TBI. The 2 groups of subjects were systematically recruited rather than randomized. The healthy group was recruited from a population who volunteered for a research imaging study, whereas the patients with mild TBI were recruited from a population who were seen with complaints of cognitive fatigue by a community neurologist. Although the same SPECT scanner was assigned to a single person for the resting and activation studies, healthy controls and patients were scanned on different scanners, possibly inducing a systematic bias. We therefore did not compare baseline $\mathrm{rCBF}$ or activation $\mathrm{rCBF}$, because of the possibility of bias due to the different scanners used. Activated $\mathrm{rCBF}$ was generated by subtraction of resting $\mathrm{rCBF}$ from activated $\mathrm{rCBF}$ and was relatively independent of bias. Another limitation of the present study was the lack of comparison between subjective symptoms and objective findings. We used PASAT score as the only measure of cognitive function. The results of PASAT serve as an objective evaluation of cognitive function but may not directly associate with subjective symptoms.

On the basis of the present findings of frontocerebellar dissociation, future studies should focus on the correlation between degree of subjective cognitive fatigue and degree of functional dissociation or should investigate a noncognitively fatigued mild-TBI cohort. In addition, longterm follow-up of the same population is needed to assess the study phenomenon over time and in response to treatment. Future studies should also investigate if cognitive fatigue in other conditions is related to a similar phenomenon and should include comparisons to other imaging modalities such as ${ }^{18} \mathrm{~F}$-FDG PET and diffusion tensor MRI.

\section{CONCLUSION}

Statistical analyses of rCBF demonstrated a different pattern of activation between patients with mild TBI and healthy control subjects. Patients with mild TBI showed dynamic changes in supratentorial rCBF during PASAT, with larger areas of activation bilaterally in the dorsolateral prefrontal cortex and larger areas of suppression in the occipital and parietal cortices.

Activation in the cerebellar cortex was prominent in healthy controls and correlated significantly with PASAT performance scores in control subjects. Patients with mild TBI showed significant correlations in the inferior frontal and superior temporal cortices instead, suggesting a compensatory mechanism. This frontocerebellar dissociation in patients with mild TBI may explain the cognitive impairment and cognitive fatigue in the chronic recovery phase of mild TBI.

\section{REFERENCES}

1. Alexander MP. Mild traumatic brain injury: pathophysiology, natural history, and clinical management. Neurology. 1995;45:1253-1260.

2. Gronwall D, Wrightson P. Delayed recovery of intellectual function after minor head injury. Lancet. 1974;2:605-609.

3. Tombaugh TN. A comprehensive review of the Paced Auditory Serial Addition Test (PASAT). Arch Clin Neuropsychol. 2006;21:53-76. 
4. Vanderploeg RD, Curtiss G, Belanger HG. Long-term neuropsychological outcomes following mild traumatic brain injury. J Int Neuropsychol Soc. 2005;11:228-236.

5. Fos LA, Greve KW, South MB, Mathias C, Benefield H. Paced Visual Serial Addition Test: an alternative measure of information processing speed. Appl Neuropsychol. 2000;7:140-146.

6. King NS. Emotional, neuropsychological, and organic factors: their use in the prediction of persisting postconcussion symptoms after moderate and mild head injuries. J Neurol Neurosurg Psychiatry. 1996;61:75-81.

7. Gronwall D, Wrightson P. Memory and information processing capacity after closed head injury. J Neurol Neurosurg Psychiatry. 1981;44:889-895.

8. Lazeron RH, Rombouts SA, de Sonneville L, Barkhof F, Scheltens P. A paced visual serial addition test for fMRI. J Neurol Sci. 2003;213:29-34.

9. Christodoulou C, DeLuca J, Ricker JH, et al. Functional magnetic resonance imaging of working memory impairment after traumatic brain injury. J Neurol Neurosurg Psychiatry. 2001;71:161-168.

10. Shiffrin RM, Schneider W. Automatic and controlled processing revisited. Psychol Rev. 1984;91:269-276.

11. Harrington DE, Malec J, Cicerone K, Katz HT. Current perceptions of rehabilitation professionals towards mild traumatic brain injury. Arch Phys Med Rehabil. 1993;74:579-586.

12. ACR practice guideline for the performance of single photon emission computed tomography (SPECT) brain perfusion and brain death studies. 2007. American College of Radiology Web site. Available at: http://www.acr.org/SecondaryMainMenuCategories/quality_safety/guidelines/nuc_med/ct_spect_brain_perfusion. aspx. Accessed May 4, 2009.

13. Lane TJ, Manu P, Matthews DA. Depression and somatization in the chronic fatigue syndrome. Am J Med. 1991;91:335-344.

14. Minoshima S, Frey KA, Koeppe RA, Foster NL, Kuhl DE. A diagnostic approach in Alzheimer's disease using three-dimensional stereotactic surface projections of fluorine-18-FDG PET. J Nucl Med. 1995;36:1238-1248.

15. Minoshima S, Koeppe RA, Frey KA, Kuhl DE. Anatomic standardization: linear scaling and nonlinear warping of functional brain images. J Nucl Med. 1994; $35: 1528-1537$.
16. Bazarian JJ, McClung J, Shah MN, Cheng YT, Flesher W, Kraus J. Mild traumatic brain injury in the United States, 1998-2000. Brain Inj. 2005;19:85-91.

17. Van Den Eeden SK, Tanner CM, Bernstein AL, et al. Incidence of Parkinson's disease: variation by age, gender, and race/ethnicity. Am J Epidemiol. 2003;157: 1015-1022.

18. Stulemeijer M, van der Werf S, Bleijenberg G, Biert J, Brauer J, Vos PE. Recovery from mild traumatic brain injury: a focus on fatigue. J Neurol. 2006; 253:1041-1047.

19. Cicerone KD. Clinical sensitivity of four measures of attention to mild traumatic brain injury. Clin Neuropsychol. 1997;11:266-272.

20. Madigan NK, DeLuca J, Diamond BJ, Tramontano G, Averill A. Speed of information processing in traumatic brain injury: modality-specific factors. $J$ Head Trauma Rehabil. 2000;15:943-956.

21. Lockwood AH, Linn RT, Szymanski H, Coad ML, Wack DS. Mapping the neural systems that mediate the Paced Auditory Serial Addition Task (PASAT). J Int Neuropsychol Soc. 2004;10:26-34.

22. Matthews MA, Carey ME, Soblosky JS, Davidson JF, Tabor SL. Focal brain injury and its effects on cerebral mantle, neurons, and fiber tracks. Brain Res. 1998;794:1-18.

23. Spanos GK, Wilde EA, Bigler ED, et al. Cerebellar atrophy after moderate-tosevere pediatric traumatic brain injury. AJNR Am J Neuroradiol. 2007;28:537-542.

24. Smith EE, Jonides J. Storage and executive processes in the frontal lobes. Science. 1999;283:1657-1661.

25. Lombardi WJ, Woolston DJ, Roberts JW, Gross RE. Cognitive deficits in patients with essential tremor. Neurology. 2001;57:785-790.

26. Desmond JE, Chen SH, DeRosa E, Pryor MR, Pfefferbaum A, Sullivan EV. Increased frontocerebellar activation in alcoholics during verbal working memory: an fMRI study. Neuroimage. 2003;19:1510-1520.

27. Goethals I, Audenaert K, Jacobs F, et al. Blunted prefrontal perfusion in depressed patients performing the Tower of London task. Psychiatry Res. 2005; 139:31-40.

28. Audenaert K, Goethals I, Van Laere K, et al. SPECT neuropsychological activation procedure with the Verbal Fluency Test in attempted suicide patients. Nucl Med Commun. 2002;23:907-916. 